\title{
Sensitivity and elasticity analysis of a Lur'e system used to model a population subject to density-dependent reproduction
}

\author{
Eric Alan Eager \\ University of Wisconsin - La Crosse \\ Richard Rebarber \\ University of Nebraska - Lincoln
}

\begin{abstract}
Sensitivity and elasticity analyses have become central to the analysis of models in population biology and ecology. While much work has been done applying sensitivity and elasticity analysis to study densityindependent (linear) matrix and integral projection models, little work has been done to study the sensitivity and elasticity of density-dependent models, especially integral projection models. In this paper we derive sensitivity and elasticity formulas for the equilibrium population $n^{*}$ of a structured population modeled by a Lur'e system, which consists of a linear system plus a nonlinearity modeling density-dependent fecundity. Sensitivity and elasticity formulas are easy to interpret ecologically, and we apply these formulas to published models for Chinook Salmon and Platte thistle (Cirsium canescens). In the C. canescens example we show that models with identical equilibrium populations can have sensitivities that are an order-of-magnitude apart, depending on the functional form for the nonlinearity.

Keywords: Sensitivity Analyses, Elasticity Analyses, Population Dynamics, Integral Projection Models, Lur'e Systems, Equilibiria
\end{abstract}

\section{Introduction}

Sensitivity and elasticity analyses have become ubiquitous in the analysis of models in population biology and ecology, beginning with Caswell [5] on the sensitivity of the asymptotic population growth rate $\lambda$ for linear population projection matrix models (PPMs, also see Caswell [7]). Easterling et al. [21] provided analogous formulas for the sensitivity and elasticity of $\lambda$ for linear integral projection models (IPMs, also

\footnotetext{
${ }^{1}$ Corresponding author. Email: eeager@uwlax.edu
} 
see [23], 25]). Sensitivity and elasticity of $\lambda$ in these cases is an application of linearization techniques combined with spectral analysis, and describes the effects of small changes in model parameters on asymptotic growth rate. These sensitivity and elasticity analyses are important in making management and conservation decisions about ecosystems, and thus have been explored in the cases where one is either not working with a deterministic linear model and/or one is instead interested in the sensitivity of something other than the longterm growth rate. For example, one may be interested in the sensitivities and elasticities of the stochastic growth rate $\lambda_{s}$ [45], 29], the population size in a stably growing population [30], a stable equilibrium in a density-dependent environment [27], [28], [47], [9], [11], 12], the growth rate for models accounting for varying sex ratios [31], some measurement of transient dynamics [29], 10], [46], [43], [16], or invasion speed [8], [35].

In many single-species structured models in population ecology, it is assumed that density-dependent feedbacks influence survival and/or reproduction between individuals as a result of competition for food, space, or other resources [14], [39], [15], [11], 12], 36]. Density-dependent feedbacks lead to models that are nonlinear, which often predict that the population converges to an equilibrium as $t \rightarrow \infty$, rather than having an asymptotic constant growth rate [14], [9], [12], 37], [44], [18]. There have been some recent studies performing sensitivity and elasticity analyses for deterministic density-dependent population models [28], [47], 9] , [12]. These studies have thus far been restricted to finite dimensional models, often using matrix calculus calculations. In recent years, many population models in the literature have been IPMs (see, for example, [38], [23], [24], [16], 13], [17], [19], [20], 25]), and a matrix analysis is not possible for these systems. Furthermore, the recent results of Caswell [12], while true for general nonlinear PPMs, are often difficult to interpret from an ecological point of view. This is in contrast to the relatively straightforward interpretation of the sensitivity (and thus elasticity) of the population growth rate $\lambda$ for linear population models, which has contributed to its widespread use throughout the theoretical ecology community. In this paper, we consider a useful class of nonlinear systems, called Lur'e systems, for which the sensitivity analysis of the asymptotic population can be straightforwardly interpreted ecologically.

Lur'e systems have been used to study the dynamics of plant and fish populations (see, for example, [38], [37], [44], [18]). These systems are of the form

$$
n_{t+1}=A n_{t}+b f\left(c^{T} n_{t}\right)
$$

Here $n_{t}$ is the population at time $t \in \mathbb{N}, A$ describes density-independent (linear) survival and growth, $f$ is a nonlinear function describing density dependence in reproduction, $b$ is the distribution of newborns, and $c^{T} n_{t}$ models the density of possible newborns at time $t$. Given some ecologically realistic assumptions on the nonlinear function $f$, Townley et al. [44], Rebarber et al. [37], Eager et al. [18], Franco et al. [26], Eager [20] and Bill et al. 4] have established the existence of a globally asymptotically stable equilibrium population $n^{*}$ 
under general conditions on $(A, b, c)$. Smith and Thieme [40] have provided comprehensive results concerning both the attractors and persistence of a more general system. A closed-form expression for the equilibrium $n^{*}$ exists in terms of $(A, b, c)$ and $f$. In this paper we show how small changes in model parameters affect the equilibrium population, as well as the "effective" equilibrium density $\tilde{N}$. We obtain expressions for the sensitivity and elasticity of $n^{*}$ and $\tilde{N}$ to changes in $(A, b, c)$ and $f$ that are rather straightforward from an ecological point of view.

We first start by providing an overview of the current theory of the long-term behavior of populations modeled with Lur'e systems and how the assumptions needed to achieve these results are reasonable ecologically. We then derive the formula for the sensitivity of $n^{*}$ and $\tilde{N}$ to changes in the linear data $(A, b, c)$ and interpret these formulas ecologically. We do this both for PPMs and IPMs. Finally, we illustrate the utility of our analysis first by applying it to a simple nonlinear population projection matrix model for Chinook Salmon found in Morris and Doak [34] and analyzed in Townley et al. [44]. We find that the equilibrium age class that is the most sensitive to changes in the model parameters is the first age class, and that the equilibrium population is most sensitive to the probability of transitioning from the first age class to the second. We then use a nonlinear integral projection model for Platte thistle (Cirsium canescens) from [38] and [16] to show how different functional forms for the nonlinearity $f$ can elicit identical conclusions with regard to the size and structure of the equilibrium population $n^{*}$, but can predict drastically different sensitivities of this equilibrium to small perturbations in the linear data $(A, b, c)$. Using a power function for $f$ elicits an equilibrium population $n^{*}$ that is less robust with respect to changes in $(A, b, c)$ than a Michaelis-Menten function yielding the same $n^{*}$, even though the nonlinearity that would have been chosen if we used AIC criterion alone is the power function [2], [16]. This complements the results in Eager et al. [16], which showed that the transient dynamics elicited in the model using a power function for $f$ are much more pronounced than those elicited in the model using a Michaelis-Menten function.

\section{The Model}

For PPMs, the population $n_{t}$ is a non-negative vector in $\mathbb{R}^{n}$, while for IPMs we will consider populations which are non-negative vectors in $L^{1}(L, U)$, the space of integrable functions on the interval $(L, U)$. Let $X$ denote either $\mathbb{R}^{n}$ or $L^{1}(L, U)$, depending on which setting we are working with. We say that a population in $\mathbb{R}^{n}$ is non-negative if all entries are non-negative. We say that a population in $L^{1}(L, U)$ is non-negative if it is non-negative on $(L, U)$ almost everywhere. We let $X^{+}$denote the set of non-negative elements of X. Because biological populations are (at least) non-negative, $X^{+}$is the natural setting to work in. The space $X^{+}$is sometimes called the state space for the system. We say that an operator $A: X \rightarrow X$ is non-negative if $A u$ is non-negative for any non-negative $u$. 
We start with a general nonlinear population model of the form

$$
n_{t+1}=M\left(n_{t}\right),
$$

where the sequence $\left\{n_{t}\right\}_{t=0}^{\infty}$ evolves in $X^{+}$. We now motivate our use of the Lur'e system (1.1). We assume that density dependence enters into the model in one (or both) of two ways: fecundity and recruitment. Both of these processes are in the reproductive stage of the life-cycle. Therefore, we follow the approach of [14], 37], 44], 17] and [40] and decompose the operator $M$ into the sum of two operators $M=A+B$, where $A$ is linear and models survival and movement between stages, and $B$ is nonlinear and models reproduction. In those papers it is further assumed that the stage of a juvenile is independent of its parent's stage. For example, in the case of a plant population modeled by (1.1), $b$ is the distribution of juvenile plants and $c^{T}$ is the functional such that $c^{T} n$ is the abundance of seeds produced by the population $n$. The nonlinearities are modeled by a function $k:[0, \infty) \rightarrow[0, \infty)$ such that $k(x)$ is the density of juveniles that are a result of $x$ possible recruits (e.g. seeds or seedlings), or a function $h:[0, \infty) \rightarrow[0, \infty)$ such that $h(x)$ is the total number of possible recruits (seeds or seedlings) that result from $x$ possible reproductive events. If one includes both of these nonlinearities, $B n=b k\left(h\left(c^{T} n\right)\right)=: b f\left(c^{T} n\right)$. With these, one can write (2.1) as (1.1).

We assume that the linear data $(A, b, c)$ satisfies the following realistic ecological assumptions from [37] and [44]:

(E1) If $X=\mathbb{R}^{n}$, then $A \in \mathcal{L}(X)$ is a non-negative matrix with spectral radius $r(A)<1$; if $X=$ $L^{1}(L, U)$, then

$$
(A u)(x)=\int_{L}^{U} P(x, y) u(y) d y,
$$

with $P \in C\left([L, U]^{2}\right), P \geq 0$, and $r(A)<1$.

(E2) $b$ is a non-negative vector;

(E3) If $X=\mathbb{R}^{n}$, then $c^{T}: X^{+} \rightarrow \mathbb{R}^{+}$is such that $A+p b c^{T}$ is primitive for every $p>0$. If $X=L^{1}(L, U)$, then $c^{T}: X^{+} \rightarrow \mathbb{R}^{+}$is a non-negative bounded functional, i.e.

$$
c^{T} n \geq 0, \quad \text { for all } n \geq 0 .
$$

Biologically, (E1) prevents the existence of immortal individuals (i.e. individuals with infinite life expectancy). (E2) states that the distribution of juveniles lives in the same space as the population itself. (E3) states that the production of possible recruits is nonnegative in the $X=L^{1}(L, U)$ case, while in the $\mathbb{R}^{n}$ case there exists a $t>0$ where the matrix $\left(A+p b c^{T}\right)^{t}$ is strictly positive for all $p>0$. The former assumption is the minimum assumption necessary for an IPM, while the latter is one that is imposed on many of the PPMs in 
the literature [41]. The positivity of $\left(A+p b c^{T}\right)^{t}$ for some $t$ ensures individuals in every stage can eventually contribute to individuals in every other stage.

It is often convenient to write $f$ as the product of the establishment probability $g$ and the number of possible recruits, i.e.

$$
f(y)=g(y) y
$$

where $g \in C(0, \infty)$ and is assumed to be a decreasing continuously differentiable function on $(0, \infty)$. We note that under these conditions, $y g^{-1}(y)$ is also continuously differentiable. We define

$$
g_{0}:=\lim _{y \rightarrow 0^{+}} g(y)
$$

and

$$
g_{\infty}:=\lim _{y \rightarrow \infty} g(y)
$$

to be the upper and lower bounds on the establishment probability of potential recruits. If $f$ is increasing, concave down, with $f(0)=0$, the long-term dynamics of (1.1) are determined by the relationship between the linear data $(A, b, c)$ and the nonlinearity $f$. The stability radius of $(A, b, c)$, denoted by $p_{e}$, is the smallest positive number $p$ such that the linear operator $A+p b c^{T}$ has spectral radius equal to 1 . It can be shown that

$$
p_{e}=\left(c^{T}(I-A)^{-1} b\right)^{-1}
$$

(see [33]). Stability results for (1.1) can be summarized in the following theorem from [37] and [44]:

Theorem 2.1. Assume that $\left\{n_{t}\right\}_{t=0}^{\infty} \subset X^{+}$solves (1.1) and the assumptions (E1), (E2) and (E3) hold, with $f$ increasing, concave down, with $f(0)=0$, then

1) If $p_{e}>g_{0}$, then the zero vector is a globally stable equilibrium for (1.1) in the sense that for every $n_{0}$ in $X^{+}$

$$
\lim _{t \rightarrow \infty} n_{t}=0
$$

Furthermore, for every $\epsilon>0$, there exists $\delta>0$ such that $\left\|n_{0}\right\| \leq \delta$ implies $\left\|n_{t}\right\| \leq \epsilon$ for all $t \in \mathbb{N}$.

2) If $p_{e} \in\left(g_{\infty}, g_{0}\right)$ then there exists $y^{*}$ which satisfies

$$
f\left(y^{*}\right)=p_{e} y^{*}
$$

(equivalently $g\left(y^{*}\right)=p_{e}$.). The vector $n^{*} \in X^{+}$given by

$$
n^{*}=p_{e} y^{*}(I-A)^{-1} b
$$


is a globally asymptotically stable equilibrium of (1.1) on $X^{+} \backslash\{0\}$, i.e.

$$
\lim _{t \rightarrow \infty} n_{t}=n^{*}
$$

and for every $\epsilon>0$, there exists $\delta>0$ such that $\left\|n_{0}-n^{*}\right\| \leq \delta$ implies $\left\|n_{t}-n^{*}\right\| \leq \epsilon$ for all $n \in \mathbb{N}$.

3) If $p_{e}<g_{\infty}$, then there exists $n_{0} \in X^{+}$such that $\limsup _{t \rightarrow \infty}\left\|n_{t}\right\|=\infty$.

We will assume for the purposes of this paper that $g_{\infty}=0$, i.e. as the abundance of possible recruits becomes arbitrarily large, the probability that any one of these possible recruits establishes is arbitrarily small.

Theorem 2.1 establishes the global asymptotic stability of the equilibrium vector $n^{*}=p_{e} y^{*}(I-A)^{-1} b$ in the case where $p_{e}<g_{0}$ and $f$ is increasing and concave down. If $f$ is not increasing (like the Ricker function $f(x)=\alpha x e^{-\beta x}$ used frequently in the fisheries sciences, for example), the results in [17], [44] and [40] provide some conditions for asymptotic stability of $n^{*}$ in the cases where global asymptotic stability cannot be achieved. Specifically, if $f=\alpha x e^{-\beta x}$, then $n^{*}$ is globally asymptotically stable for $p_{e} \in\left(\alpha e^{-2}, \alpha\right)$, while if $p_{e} \in\left(0, \alpha e^{-2}\right)$ asymptotic stability depends on the spectral radius of the linearized operator

$$
A+f^{\prime}\left(c^{T} n^{*}\right) b c^{T}=A+\frac{p_{e}}{\beta} \log \left(\frac{\alpha}{p_{e}}\right) b c^{T} .
$$

For the remainder of this paper we will assume that at least asymptotic stability of $n^{*}$ holds, and the perturbations to $(A, b, c)$ are such that the resulting equilibrium population is also asymptotically stable.

\section{Results}

In this section we derive the formula for the sensitivity of the equilibrium population $n^{*}$ and the "effective" equilibrium populations $\tilde{N}$ with respect to changes in the linear data $(A, b, c)$. An effective equilibrium $\tilde{N}$ is a weighted average of the population sizes within the various stages of $n^{*}$. In particular, we look at these sensitivities when $n^{*}>0$ is a positive equilibrium population (that is, $g_{0}>p_{e}$ in Theorem 2.1). We then use these sensitivity formulas to find, in the standard way, corresponding elasticity formulas to help us correct for differences we see in the relative sizes of parameter values we consider.

\subsection{Sensitivity of $n^{*}$ to perturbations in $(A, b, c)$}

We first consider changes in the equilibrium population $n^{*}$ with respect to changes in the survival/growth operator $A$. Let $\Delta A$ be a bounded operator, and consider the perturbed operator $A_{\epsilon}:=A+\epsilon \Delta A$ for $\epsilon>0$. For this perturbation to make sense ecologically, we need $\epsilon$ to be small enough such that $r\left(A_{\epsilon}\right)<1$, so that 
$A_{\epsilon}$ is still a survival/growth operator. Since $\Delta A$ is bounded, this is always true for small enough $\epsilon$, since by the stability radius theory in [33],

$$
r\left(A_{\epsilon}\right)<1 \quad \text { for }\|\epsilon \Delta A\|<\left(c^{T}(I-A)^{-1} b\right)^{-1} .
$$

We say the sensitivity of $n^{*}(A)$ to $\Delta A$ is

$$
S(\Delta A):=\lim _{\epsilon \rightarrow 0} \frac{n^{*}(A+\epsilon \Delta A)-n^{*}(A)}{\epsilon} .
$$

If $h$ is a function of $A$, then we write

$$
D_{\Delta A} h:=\lim _{\epsilon \rightarrow 0} \frac{h(A+\epsilon \Delta A)-h(A)}{\epsilon} .
$$

When $\|\Delta A\|=1, D_{\Delta A} h$ is the directional derivative of $h$ in the direction of $\Delta A$.

The resulting change in $n^{*}=p_{e} y^{*}(I-A)^{-1} b$ can be decomposed as follows:

$$
n^{*}(A+\epsilon \Delta A)-n^{*}(A)=\Delta\left(p_{e} y^{*}\right)(I-A)^{-1} b+p_{e} y^{*} \Delta\left((I-A)^{-1} b\right)+\Delta\left(p_{e} y^{*}\right) \Delta\left((I-A)^{-1} b\right),
$$

since a change in $A$ also affects $p_{e}$ and $y^{*}$. Changes in $n^{*}$ are composed of changes to both the long-term reproduction $p_{e} y^{*}$ and long-term stage distribution $(I-A)^{-1} b$. We first explore changes in $p_{e} y^{*}$ with respect to changes in $A$.

We express the term $p_{e} y^{*}$ as a function of $p_{e}$ : using (2.3) and (2.1), we see that

$$
p_{e} y^{*}=p_{e} g^{-1}\left(p_{e}\right)
$$

Since $y g^{-1}(y)$ is continuously differentiable,

$$
\Delta\left(p_{e} y^{*}\right)=\left(p_{e} g^{-1}\left(p_{e}\right)\right)^{\prime} \Delta\left(p_{e}\right)+o\left(\Delta p_{e}\right)
$$

Since $p_{e}=\left(c^{T}(I-A)^{-1} b\right)^{-1}$ we can calculate $\Delta\left(p_{e}\right)$ exactly as follows:

$$
\Delta\left(p_{e}\right)=\frac{1}{c^{T}(I-(A+\epsilon \Delta A))^{-1} b}-\frac{1}{c^{T}(I-A)^{-1} b}=\epsilon \frac{-c^{T}(I-(A+\epsilon \Delta A))^{-1} \Delta A(I-A)^{-1} b}{c^{T}(I-(A+\epsilon \Delta A))^{-1} b c^{T}(I-A)^{-1} b},
$$

where $(I-(A+\epsilon \Delta A))^{-1}$ is defined because of our assumption that $r(A+\epsilon \Delta A)<1$. This shows that $\Delta p_{\epsilon}=\mathcal{O}(\epsilon)$, so

$$
\Delta\left(p_{e} y^{*}\right)=\left(p_{e} g^{-1}\left(p_{e}\right)\right)^{\prime} \Delta\left(p_{e}\right)+o(\epsilon)
$$

Dividing (3.1) by $\epsilon$ and taking the limit as $\epsilon \rightarrow 0$,

$$
\left(D_{\Delta A}\right) p_{e}=\frac{-c^{T}(I-A)^{-1} \Delta A(I-A)^{-1} b}{\left(c^{T}(I-A)^{-1} b\right)^{2}}=-\left(p_{e}\right)^{2} w^{T}(\Delta A) v,
$$


where $w^{T}:=c^{T}(I-A)^{-1}$ and $v:=(I-A)^{-1} b$ are the left and right eigenvectors, respectively, of the operator $A+p_{e} b c^{T}$, with eigenvalue $\lambda=1$. Using a similar calculation we have that

$$
\left(D_{\Delta A}\right)(I-A)^{-1} b=(I-A)^{-1} \Delta A v .
$$

Therefore,

$$
\left(D_{\Delta A}\right) n^{*}=\frac{d p_{e} g^{-1}\left(p_{e}\right)}{d p_{e}}\left(-\left(p_{e}\right)^{2} w^{T}(\Delta A) v\right) v+p_{e} g^{-1}\left(p_{e}\right)(I-A)^{-1}(\Delta A) v
$$

Now suppose, as in the PPM case, that $X=\mathbb{R}^{n}$ and $\Delta A=\Delta A_{i j}$, the matrix will all zero entries except for a 1 in the $(i, j)^{\text {th }}$ entry. In this case we denote $D_{\Delta A}$ as $D_{i j}$. We write $w^{T}=\left[w_{1}, w_{2}, \ldots w_{n}\right]$ and $v=\left[v_{1}, v_{2}, \ldots v_{n}\right]^{T}$, so the sensitivity of $n^{*}$ to a perturbation of the $(i, j)^{\text {th }}$ element of $A$ is

$$
D_{i j} n^{*}:=\left(D_{\Delta A_{i j}}\right) n^{*}=\frac{d p_{e} g^{-1}\left(p_{e}\right)}{d p_{e}}\left(-\left(p_{e}\right)^{2} w_{i} v_{j}\right) v+p_{e} g^{-1}\left(p_{e}\right)\left((I-A)^{-1}\right)_{i} v_{j},
$$

where $\left.(I-A)^{-1}\right)_{i}$ is the $i$ th column of the matrix $(I-A)^{-1}$.

Notice that the sensitivity of the equilibrium population $n^{*}$ to a change in $A$ is the sum of the scaled change as a result of changes in equilibrium reproduction (given by $p_{e} y^{*}=p_{e} g^{-1}\left(p_{e}\right)$ ) multiplied by the equilibrium population structure (given by $v=(I-A)^{-1} b$ ) and the change due to changes in equilibrium population structure $v$ multiplied by the original equilibrium reproduction $p_{e} y^{*}$. It is easy to see that

$$
\left(p_{e} g^{-1}\left(p_{e}\right)\right)^{\prime} g^{\prime}\left(y^{*}\right)=f^{\prime}\left(y^{*}\right)
$$

so if $f$ is increasing, $p_{e} g^{-1}\left(p_{e}\right)$ is a decreasing function of $p_{e}$ (since $g$ is decreasing), and hence the sensitivity (3.2) is positive. If $f$ is not increasing, e.g. $f$ is given by the Ricker function $f(x)=\alpha x e^{-\beta x}$, this is not necessarily the case due to overcompensatory density dependence [42].

We now consider the sensitivity of $n^{*}$ to perturbations $\epsilon \Delta b$ of the vector $b$ from (1.1). In order for the perturbed model to make sense ecologically, $\epsilon$ and $\Delta b$ should be such that $b+\epsilon \Delta b$ is a non-negative vector. Assuming this is the case one can show that, via an analogous calculation to that for $D_{\triangle A} n^{*}$, the sensitivity of the equilibrium population $n^{*}$ to a perturbation of the vector $b$ is

$$
D_{\Delta b} n^{*}=\frac{d p_{e} g^{-1}\left(p_{e}\right)}{d p_{e}}\left(-\left(p_{e}\right)^{2} w^{T} \Delta b\right) v+p_{e} g^{-1}\left(p_{e}\right)(I-A)^{-1} \Delta b .
$$

We can similarly consider the sensitivity of $n^{*}$ to a perturbation $\epsilon \Delta c^{T}$ of the functional $c^{T}$. When $X=\mathbb{R}^{n}$, we can consider a perturbation to the $i$ th component of $c$, so $\Delta c_{i}$ is a vector with an 1 in the $i$ th component and 0 everywhere else; this perturbation models the event where the per-capita production of possible juveniles is increased in the $i$ th stage. Employing a similar calculation to the ones for (3.2) and (3.4), one can show that the sensitivity of the equilibrium population $n^{*}$ to a perturbation of $c^{T}$ is

$$
D_{\Delta c} n^{*}=-\frac{d p_{e} g^{-1}\left(p_{e}\right)}{d p_{e}}\left(p_{e}\right)^{2}\left(\left(\Delta c^{T}\right) v\right) v
$$


When $\Delta c^{T}=\Delta c_{i}^{T}$,

$$
D_{\Delta c_{i}} n^{*}=\frac{d p_{e} g^{-1}\left(p_{e}\right)}{d p_{e}}\left(-\left(p_{e}\right)^{2} v_{i}\right) v .
$$

Notice that, since the linear equilibrium population structure $v=(I-A)^{-1} b$ does not depend on $c^{T}$, the sensitivity $D_{\Delta_{c}} n^{*}$ comes only from changes in the nonlinear equilibrium reproduction.

Like the sensitivity of the growth rate $\lambda$ for linear models, all of these sensitivity formulas are in terms of the right and/or left eigenvectors $w, v$ of the operator $A+p_{e} b c^{T}$ at equilibrium (i.e. with eigenvalue equal to 1 ). What makes these formulas more straightforward than those derived for other sensitivities of equilibria (for example, [11], [12]) are the explicit formulae for the eigenvectors $w^{T}=c^{T}(I-A)^{-1}$ and $v=(I-A)^{-1} b$ and the explicit dependence of $n^{*}$ on the stability radius $p_{e}=\left(c^{T}(I-A)^{-1} b\right)^{-1}$, which are features of the Lur'e system. The right eigenvector $w^{T}$ represents a scaled version of the "net reproduction" vector [14], the left eigenvector $v$ represents a scaled version of the "stable stage distribution" vector [7], and $p_{e}$ (which is found via a straightforward formula in terms of $(A, b, c))$ determines the size of the equilibrium population via the nonlinearity $f$.

\subsection{Sensitivities for Integral Projection Models}

To apply the formulas in the previous section to IPMs, we first need to discuss $(A, b, c)$ for nonlinear IPMs of the form (1.1). Recall that by assumption (E1), $P$ is a continuous function on $[L, U]^{2}$, and defines the survival/growth integral operator $A$ on $L^{1}(L, U)$ by (2.2). The juvenile distribution vector $b \in L^{1}(L, U)$ has an associated probability density function $b(x)$ for the initial stage of newly reproduced members. Let $c(x)$ be a bounded function on $(L, U)$ representing potential reproductive output (for instance, seeds) as a function of the stage variable $x \in(L, U)$, and let $c^{T}$ be the bounded functional on $L^{1}(L, U)$ be defined by

$$
c^{T} u=\int_{L}^{U} c(y) u(y) d y .
$$

It will be clear from the context whether $b$ and $c$ denote the vector or the function of interest, so we use the same letter for both. We assume that $b$ and $c$ are continuous functions on $[L, U]$, and recall that $P$ is continuous on $[L, U]^{2}$.

The formulas (3.2), (3.4) and (3.5) all hold in $L^{1}(L, U)$ provided that $\Delta A$ is a bounded operator on $L^{1}(L, U)$, $\Delta b \in L^{1}(L, U)$ and $\Delta c$ is a bounded functional on $L^{1}(L, U)$. However, we want to be able to consider unbounded perturbations of $A$, as in Ellner and Rees [23]. Specifically, we seek the analog of a matrix perturbation $A_{i j}$ in the infinite dimensional space $L^{1}(L, U)$. To do so, we will follow Ellner and Rees [23] and fix $\left(x_{0}, y_{0}\right) \in(L, U)^{2}$. Consider the Dirac delta function $\delta\left(x-x_{0}, y-y_{0}\right)$ for $x_{0}, y_{0} \in(L, U)$. An operator 
on $L^{1}(L, U)$ which is analogous to $\Delta A_{i j}$ is, formally,

$$
\left(\Delta A_{\left(x_{0}, y_{0}\right)} u\right)(x)=\int_{L}^{U} \delta\left(x-x_{0}, y-y_{0}\right) u(y) d y=\delta\left(x-x_{0}\right) u\left(y_{0}\right) .
$$

However, this operator has domain which is smaller than $L^{1}(L, U)$, since $u$ is in the domain of $\Delta A_{\left(x_{0}, y_{0}\right)}$ only if $u\left(y_{0}\right)$ is defined. Furthermore, the range of $\Delta A_{\left(x_{0}, y_{0}\right)}$ is not in $L^{1}(L, U)$. In Appendix B we treat $\Delta A_{\left(x_{0}, y_{0}\right)}$ as a distribution, and show that

$$
\left(D_{\Delta A_{\left(x_{0}, y_{0}\right)}}\right) n^{*}=-\frac{d p_{e} g^{-1}\left(p_{e}\right)}{d p_{e}}\left(p_{e}\right)^{2} w\left(x_{0}\right) v\left(y_{0}\right) v+p_{e} g^{-1}\left(p_{e}\right)\left(\delta\left(\cdot-x_{0}\right)+(I-A)^{-1} P\left(\cdot, x_{0}\right)\right) v\left(y_{0}\right)
$$

in the sense of distributions.

While $D_{\Delta A_{\left(x_{0}, y_{0}\right)}}$ is not a bounded operator, we can still discuss the change in $n^{*}$ when $A$ changes to $A+\epsilon \Delta A_{\left(x_{0}, y_{0}\right)}$. Formally, this change is approximately (to first order in $\epsilon$ )

$$
\epsilon\left(-\frac{d p_{e} g^{-1}\left(p_{e}\right)}{d p_{e}}\left(p_{e}\right)^{2} w\left(x_{0}\right) v\left(y_{0}\right) v+p_{e} g^{-1}\left(p_{e}\right)\left(\delta\left(\cdot-x_{0}\right)+(I-A)^{-1} P\left(\cdot, x_{0}\right)\right) v\left(y_{0}\right)\right) .
$$

While this is not a function, we can interpret this in the following way. The approximate change (to first order in $\epsilon$ ) in the size of population between $a$ and $b$ (where $L<a<b<U$ ) when $A$ changes to $A+\epsilon \Delta A_{\left(x_{0}, y_{0}\right)}$ is

$$
\left.\left.\epsilon\left(-\frac{d p_{e} g^{-1}\left(p_{e}\right)}{d p_{e}}\left(p_{e}\right)^{2} w\left(x_{0}\right) v\left(y_{0}\right)\right] \int_{a}^{b} v(x) d x+p_{e} g^{-1}\left(p_{e}\right) v\left(y_{0}\right)\left(\chi_{(a, b)}\left(x_{0}\right)+\int_{a}^{b}(I-A)^{-1} P\left(\cdot, x_{0}\right)\right) d x\right)\right),
$$

where $\chi_{(a, b)}$ is the characteristic function for the interval $(a, b)$.

We now discuss how changes in $b$ and $c^{T}$ affect $n^{*}$. Let $\Delta b \in L^{1}(L, U)$ be a perturbation vector, and assume that $\epsilon>0$ is such that $b+\epsilon \Delta b$ is also a positive function (almost everywhere). In particular, $b(x)+\epsilon \Delta b(x) \geq 0$ a.e. and

$$
\int_{L}^{U} \Delta b(x) d x=0
$$

Note that here we are only considering $\Delta b$ which are in $L^{1}(L, U)$, so the formula for the sensitivity of $n^{*}$ to perturbations in $b$ given by (3.4) makes sense for systems in $L^{1}(L, U)$.

A functional on $L^{1}(L, U)$ which is analogous to the perturbation $\Delta c_{i}^{T}$ is, in the sense of distributions,

$$
\Delta c_{y_{0}}^{T} u=\int_{L}^{U} \delta\left(x-x_{0}\right) u(y) d y=u\left(y_{0}\right)
$$

for a fixed $y_{0} \in(L, U)$. Using (3.5), and an argument similar to (but simpler than) that for changes in $A$, we see that

$$
D_{\Delta c_{x_{0}}} n^{*}=\frac{d p_{e} g^{-1}\left(p_{e}\right)}{d p_{e}}\left(-\left(p_{e}\right)^{2} v\left(x_{0}\right)\right) v
$$


Since $v$ is continuous, this sensitivity is well-defined and continuous.

\subsection{Sensitivity of the "effective" equilibrium population size $\tilde{N}$}

In this section we are interested in the sensitivity of the "effective" equilibrium population $\tilde{N}$ ([11], [12]), which is a weighted sum or integral of the total densities of individuals within the various stages of $n^{*}$, to changes in the linear data $(A, b, c)$. When this combination of population sizes is linear and $\tilde{N}$ is scalar, this effective population can be written as $d^{T} n^{*}$ for some linear functional $d^{T}$ on $X$. As an example, when $X=\mathbb{R}^{n}$ and one only cares about the total size of the equilibrium population $n^{*} \in \mathbb{R}^{n}$, then $d^{T}$ is the vector with 1 in all of its components, and $\tilde{N}=d^{T} n^{*}=\sum_{i=1}^{n} n_{i}^{*}$. If one only cared about the size of the population in the $i$ th stage, then $d^{T}$ would be a vector with a 1 in the $i$ th component and zeros everywhere, with $\tilde{N}=d^{T} n^{*}=n_{i}^{*}$. As an example when $X=L^{1}(L, U)$, the size of the population between stages $a$ and $b$ is given by

$$
d^{T} n=\int_{L}^{U} \chi_{[a, b]}(x) n(x) d x
$$

with the size of the entire population given when $\chi_{[L, U]}$ replaces $\chi_{[a, b]}$. Since $d^{T}$ is linear, to compute the sensitivities of $\tilde{N}$, we simply apply $d^{T}$ to the formulas (3.2), (3.4) and (3.5) to obtain the desired sensitivity. If the effective population is defined by $\tilde{N}=H\left(n^{*}\right)$, where $H$ is a nonlinear (Frechét) differentiable function $H: X^{+} \rightarrow \mathbb{R}^{+}$, then one would apply the chain rule to $\tilde{N}=H\left(n^{*}\right)$ to find the resulting sensitivity of $\tilde{N}$.

\subsection{Elasticity of $n^{*}$ and $\tilde{N}$}

For some purposes, we are interested in the effect of a proportional change in the data on $n^{*}$ and $\tilde{N}$. For example, survival and growth probabilities in $A$ are all going to be values between 0 and 1 , while reproductive values in $c^{T}$ may be much larger than 1, see 7]. It might not be reasonable to compare the sensitivity of $n^{*}$ to survival to the sensitivity of $n^{*}$ to fecundity, for example. In such a case, we look at the elasticity of $n^{*}$ and $\tilde{N}$ to changes in $(A, b, c)$. As usual, this can be calculated by simply multiplying the sensitivity values derived in Sections 3.1 and 3.2 by the numerical value of the term perturbed divided by that of the element for which we are taking the sensitivity. For example, in the PPM case, the elasticity of $n_{i}^{*}$, the $i$ th element of $n^{*}$ to changes in $A_{i, j}$ would be given by $A_{i, j}\left(n_{i}^{*}\right)^{-1}\left(D_{i j} n^{*}\right)_{i}$, where the vector of sensitivities $\left(D_{i j} n^{*}\right)$ is from (3.3). We will use both sensitivity and elasticity calculations to study how changes in the linear data $(A, b, c)$ affect the equilibrium population $n^{*}$ and effective total population size $\tilde{N}$ in the following two examples. 


\section{Examples}

\subsection{PPM for Chinook Salmon}

We first consider the sensitivity and elasticity of the equilibrium population $n^{*}$ for a PPM for Chinook Salmon from Morris and Doak [34], whose stability was studied in Townley et al. [44]. As in [44], we first assume a Michaelis-Menten type form $f(x)=V x(K+x)^{-1}$ for the nonlinear fecundity function and then discuss how our sensitivity analysis can be applied when $f(x)=x \exp (-\beta x)$ is a Ricker-type function.

The model is structured with five age classes, where fish with age classes 3-5 able to spawn. The linear data is

$$
A=\left[\begin{array}{ccccc}
0 & 0 & 0 & 0 & 0 \\
s_{1} & 0 & 0 & 0 & 0 \\
0 & s_{2} & 0 & 0 & 0 \\
0 & 0 & s_{3} & 0 & 0 \\
0 & 0 & 0 & s_{4} & 0
\end{array}\right], \quad b:=\left[\begin{array}{l}
1 \\
0 \\
0 \\
0 \\
0
\end{array}\right], \quad c^{T}:=\left[\begin{array}{lllll}
0 & 0 & f_{3} & f_{4} & f_{5}
\end{array}\right],
$$

where $s_{i}$ is the probability of an individual of age $i$ surviving to age $i+1$ in one time-step and $f_{i}$ the number of eggs produced by an individual of age $i$ in one time-step. We use the following parameter values from 44]:

$$
s_{1}=0.0131 \quad s_{2}=0.8 \quad s_{3}=0.7896 \quad s_{4}=0.6728
$$

and

$$
f_{3}=2.2834 \quad f_{4}=35.1099 \quad f_{5}=277.6529 .
$$

With these parameter values it follows that the stability radius $p_{e}=0.5376$. In the first case we use the Michaelis-Menten nonlinearity with $V=6$ and $K=8$, which means that $g_{0}=0.75$ and $p_{e}<g_{0}$. This, along with the fact that $A+p b c^{T}$ is primitive for all $p>0$, means that Theorem 2.1, part 2) applies. The globally asymptotically stable equilibrium $n^{*}$ in this case is given by

$$
n^{*}=p_{e} g^{-1}\left(p_{e}\right)(I-A)^{-1} b=\left(V-p_{e} K\right)(I-A)^{-1} b=\left[\begin{array}{lllll}
1.6996 & 0.0223 & 0.0178 & 0.0141 & 0.0095
\end{array}\right]^{T} .
$$

Since $s_{1}, s_{2}, s_{3}, s_{4}, f_{3}, f_{4}$ and $f_{5}$ are the only ecologically reasonable parameters in $(A, b, c)$ to perturb, we will study the sensitivity of the vector $n^{*}$ to changes in these values. Since the model is a PPM, there is no difficulty in using the formulas (3.3) and (3.5) to determine the sensitivity of $n^{*}$ in this example. We will look at the sensitivity of each stage in the equilibrium population $n^{*}$ to changes in each of these parameters. For example, the sensitivity of $n_{i}$ to changes in $s_{1}$, which is the $(2,1)$ entry of $A$, is given by

$$
D_{21} n_{i}^{*}=K\left(\left(p_{e}\right)^{2} w_{2} v_{1}\right) v_{i}+\left(V-p_{e} K\right)\left((I-A)^{-1}\right)_{2, i} v_{1} .
$$


Figure 1 shows how sensitive $n^{*}$ is to changes in the survival parameters $s_{1}, s_{2}, s_{3}$ and $s_{4}$. For all four parameters the first age class is the most sensitive age class, and the equilibrium population in each age class is most sensitive to changes in the parameter $s_{1}$, the probability of survival from age class 1 to age class 2 . These results can be seen more clearly by looking at the general sensitivity formula (3.3) and the specific sensitivity formula (4.1): The coefficient $K\left(p_{e}\right)^{2}=27.68$ is much larger than $V-p_{e} K=1.699$, meaning that the first term in (3.3) is going to be the dominant value in the sensitivity. The first term is a constant multiple of the vector $v$, which is weighted heavily towards the first stage. When $(i, j)=(2,1)$ the value $v_{1}$, the largest $v_{i}$, is in both terms, eliciting the largest sensitivity of the survival parameters $s_{i}$ for all of the age stages.

Figure 2 shows how sensitive $n^{*}$ is to changes in the egg production parameters $f_{3}, f_{4}$ and $f_{5}$. As with the survival/growth parameters we will look at the sensitivity of each stage in the equilibrium population to changes in each of these parameters. For example, the sensitivity of $n_{i}$ to changes in $f_{3}$, which is the third element of $c^{T}$, is given by

$$
D_{\Delta c_{3}} n_{i}^{*}=\frac{d p_{e} g^{-1}\left(p_{e}\right)}{d p_{e}}\left(-\left(p_{e}\right)^{2} v_{3}\right) v_{i}
$$

For all three parameters the first age class is the most sensitive, and the equilibrium population in each age class is most sensitive to changes in the parameter $f_{3}$, the number of eggs produced by individuals in age class 3. Thus, for this model, it is clear that survival from the first age class to the second is the parameter to which the population is most sensitive, and changes in any of the parameters will affect the first age class the most. The reasons for this are straightforward from the formulas (3.3) and (4.2): The sensitivity of $n^{*}$ to changes in $c_{i}$ is a constant times the vector $v$, which is most heavily-weighted towards the first age class, times $v_{i}$, which is decreasing in $i$.

To further investigate these results we also looked at the elasticity of $n^{*}$ to changes in all of the modeling parameters. The figures coinciding with these calculations are in Appendix A. When looking at the elasticity of $n^{*}$ to the various model parameters the story changes only slightly: the equilibrium population's first age class is still the equilibrium age class that is most elastic to changes in all of the model parameters, but the elasticity of $n^{*}$ to changes in $s_{2}, s_{3}$ and $s_{4}$ are much closer to that of $s_{1}$ than are the sensitivities, suggesting that the large discrepancies seen in Figure 4.1 are largely due to those in the age distribution in $n^{*}$. The elasticity of $n^{*}$ to changes in $f_{4}$ is the largest out of the $f_{i}$ 's, which is in contrast to the sensitivities, where $f_{3}$ elicited the largest sensitivity, albeit only slightly.

When one uses the Ricker function $f(x)=x \exp (-\beta x)$ (with $\alpha=1$ ) for fecundity the sensitivity of $n^{*}$ to all parameters is negative for the $\beta=0.05$ value used in Townley et al. [44], since by changing from the MichaelisMenten function to the Ricker function, we change $p_{e} g^{-1}\left(p_{e}\right)$ from $V-p_{e} K$ to $p_{e}(\beta)^{-1} \log \left(p_{e}^{-1}\right)$ and $\frac{d p_{e} g^{-1}\left(p_{e}\right)}{p_{e}}$ from $-K$ to $(\beta)^{-1}\left(1-\log \left(p_{e}^{-1}\right)\right)$. The latter change causes the first term in the sensitivity formula $D_{i j} n^{*}$ and 
the entire formula $D_{\Delta c_{i}} n^{*}$ to be negative, making both of these sensitivities negative. Thus, at equilibrium, the population predicted by the model with the Ricker function is experiencing overcompensatory density dependence, and increasing any of the population parameters will cause more density-dependent feedback, which will cause a decrease in the population's equilibrium. This is an example of the Hydra effect [1], and is a good illustration of why it is important to use the proper functional form for the nonlinearity modeling density-dependent feedbacks, as failing to do so can cause conservation or harvesting efforts to have unpredicted results.

\subsection{IPM for the Platte thistle}

For our second example we use the sensitivity developed in this paper to further study the effects of structural model uncertainty of a nonlinear IPM of the form (1.1) from Rose et al. 38] for Platte thistle (Cirsium canescens). In Eager et al. [16], we showed that structural model uncertainty can greatly influence the system's predicted transient dynamics by comparing two possible functional forms for $f$. Here we show how the sensitivity and elasticity of equilibrium populations to changes in the linear data $(A, b, c)$ are also greatly influenced by the nonlinear function $f$ used to model density-dependent fecundity. This is an important aspect to consider in ecological modeling because most ecological systems are driven by some density-dependent mechanisms, but the signal for density dependence in empirical data sets is often weak. A typical example of this is the seedling recruitment data in Fig. 1 of [38]: the data are few, very noisy, and collected over a limited range of seed densities. It is challenging to find sensible functions that fit such noisy data well, and commonly used criteria to choose among different candidate functions (such as Akaike information criterion (AIC) or Bayesian information criterion) can only provide a relative ranking of poor fitting functions.

Rose et al.'s model is of the Lur'e structure (1.1) studied in this paper, where the population $n_{t}$ is structured with respect to size. The authors in that paper use the natural logarithm of a plant's root crown diameter as a proxy for its size. The survival/growth operator $A$ is a linear integral operator of the form

$$
(A u)(x)=\int_{L}^{U} P(x, y) u(y) d y
$$

for each $u \in L^{1}[L, U]$, with $P(x, y)$ the product of the probabilities of survival, growth and not flowering, where $y$ is the size of the plant at time $t$ and $x$ is the size of the plant at time $t+1$. The functional $c^{T}$ is a linear functional of the form

$$
c^{T} u=\int_{L}^{U} c(y) u(y) d y,
$$

for each $u \in L^{1}[L, U]$, where $c(\cdot)$ is the product of the seed production, survival and flowering probabilities as a function of the size of the plant $y$ at time $t$. The vector $b$ is a probability distribution over $[L, U]$, which in this model is $[-2,5.5]$. All functional forms are available via Table 2 in [38]. 
(a)

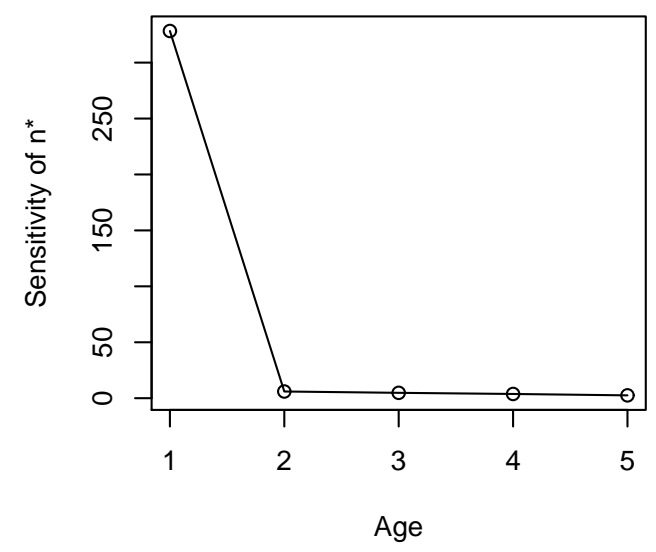

(c)

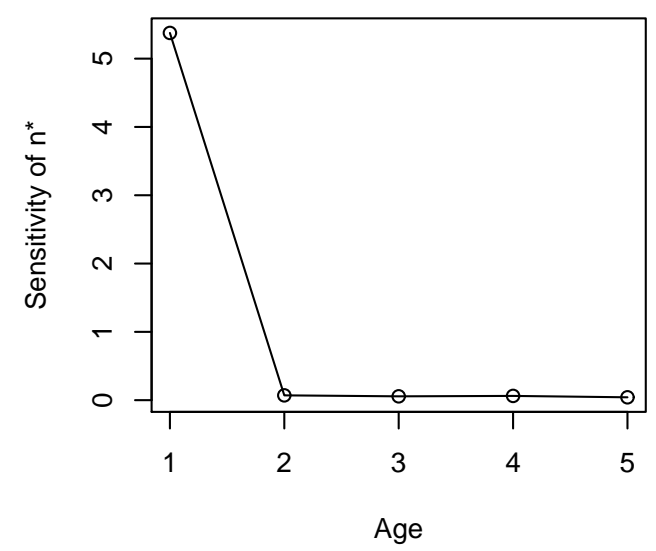

(b)

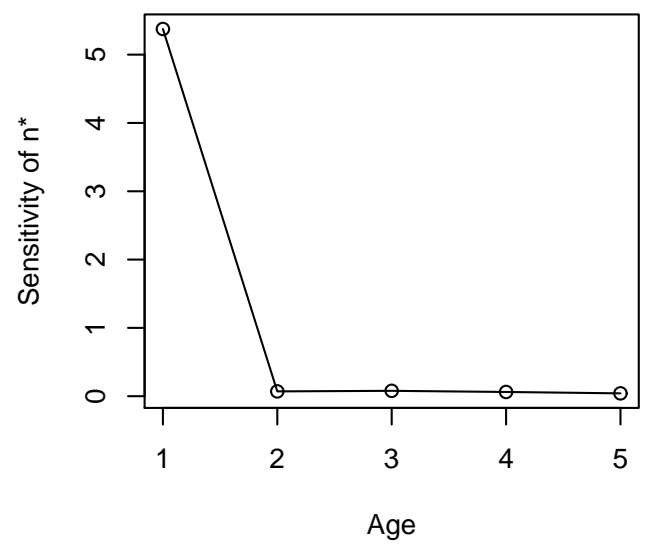

(d)

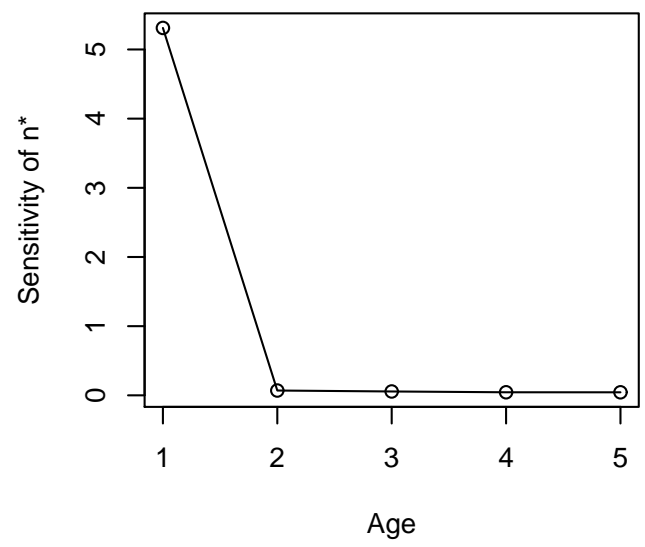

Figure 1: Sensitivity values for the equilibrium population $n^{*}$ with respect to changes in the survival parameters. The horizontal axis is the age, while the vertical axis is the sensitivity of the value of $n^{*}$ at that age to changes in the parameter (a) $s_{1}$, (b) $s_{2},(\mathrm{c}) s_{3}$ and (d) $s_{4}$. 
(a)

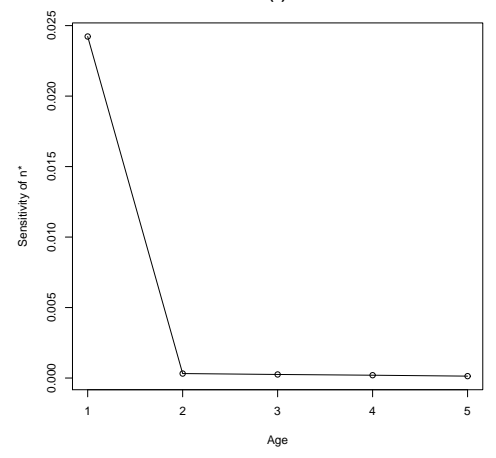

(b)

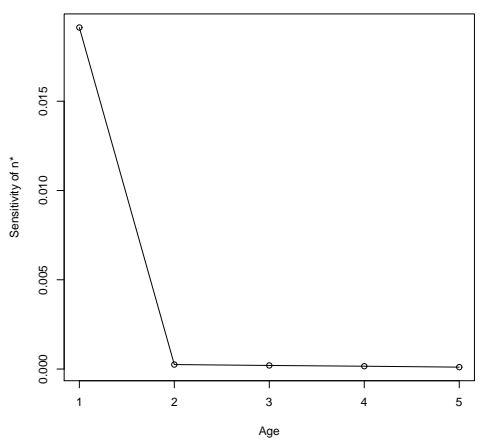

(c)

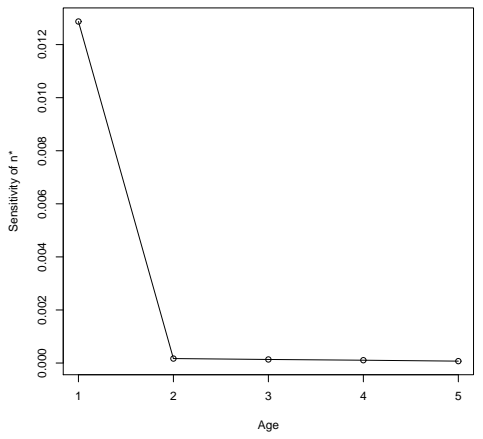

Figure 2: Sensitivity values for the equilibrium population $n^{*}$ with respect to changes in the fecundity parameters. The horizontal axis is the age variable, while the vertical axis is the sensitivity of the value of $n^{*}$ at that age variable to changes in the parameter (a) $f_{3}$, (b) $f_{4}$ and (c) $f_{5}$.

Rose et al. 38] models density-dependent seedling recruitment with a power function of the form $f_{1}(x)=x^{\nu}$, where $x$ is the density of seeds produced by the population in one time-step, $f_{1}(x)$ is the density of seedlings that result from these seeds, and the parameter $\nu$ is fit to data. If $\nu \in(0, \alpha)$ (which is essential to describe negative density-dependent dynamics using such a power function), the power function has pathological (i.e. not biologically motivated) properties which include having an unbounded derivative for low seed densities and being unbounded for large seed densities. These extreme values of $x$ are outside of the range of seed densities considered in the statistical analysis of [38]. Therefore, in [16] we derived an alternative to the power function for seedling recruitment based on biological principles that takes into account these extreme values. The resulting function $f_{2}(x)=\alpha x(\beta+x)^{-1}$ is the classical Michaelis-Menten function mentioned previously. Both of these functions satisfy the conditions of Theorem 2.1. and thus we can use the formulas derived in this paper to study the sensitivity and elasticity of $n^{*}$ to changes in linear data $(A, b, c)$, and how these sensitivities differ when one uses $f_{1}$ or $f_{2}$ for $f$. We constructed $f_{2}$ so that its resulting equilibrium population is the same as that which uses $f_{1}$ for $f$.

We will look at how changes in the survival/growth kernel $P(\cdot, \cdot)$ and changes in the seed production function $c(\cdot)$ change the equilibrium population $n^{*}$ of the two models by exploring how sensitive the equilibrium total population size $\tilde{N}=\left\|n^{*}\right\|$ and the equilibrium total seed production $\tilde{N}=c^{T} n^{*}$ is to these aformentioned changes to $A$ and $c^{T}$. For the former the effective population size is found by using $d^{T}$ defined by $\chi_{[L, U]}$ and 
(a)

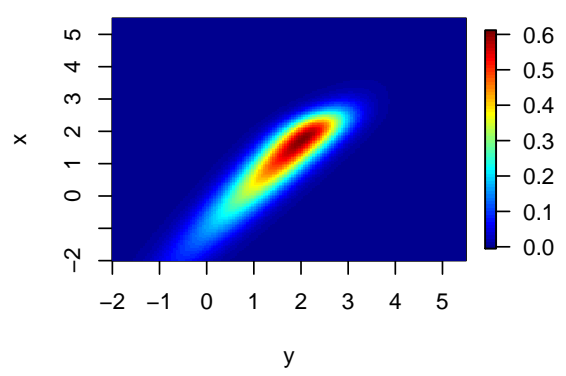

(c)

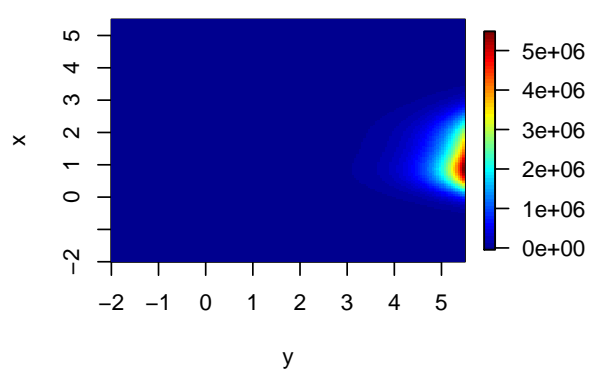

(b)

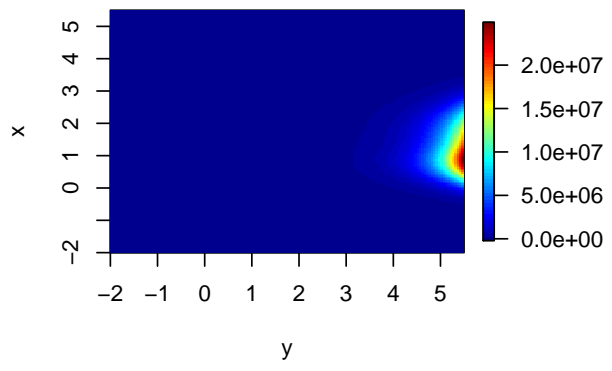

(d)

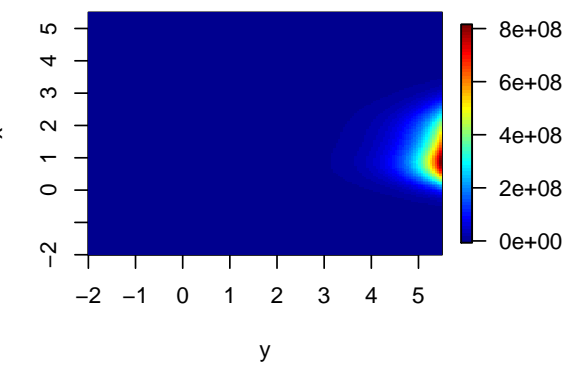

Figure 3: Illustration of the sensitivity values for the equilibrium population $n^{*}$ from Rose et al. (2005) to changes in the kernel function $P(\cdot, \cdot)$. (a) gives a graph of $P(\cdot, \cdot)$, (b) the sensitivities of $\left\|n^{*}\right\|$ to changes in $P(\cdot, \cdot)$ for the model using the power function, (c) the sensitivities of $\left\|n^{*}\right\|$ to changes in $P(\cdot, \cdot)$ for the model using the Michaelis-Menten function and (d) the sensitivities of $c^{T} n^{*}$ for the model using the power function.

the latter by $d^{T}=c^{T}$. Using (3.6) and (3.8), we see that these sensitivities are equal to $\left(D_{\Delta A_{\left(x_{0}, y_{0}\right)}}\right)\left\|n^{*}\right\|=-\frac{d p_{e} g^{-1}\left(p_{e}\right)}{d p_{e}}\left(p_{e}\right)^{2} w\left(x_{0}\right) v\left(y_{0}\right)\|v\|+p_{e} g^{-1}\left(p_{e}\right) v\left(y_{0}\right)\left\|\delta\left(\cdot-x_{0}\right)+(I-A)^{-1} P\left(\cdot, x_{0}\right)\right\|$, $\left(D_{\Delta A_{\left(x_{0}, y_{0}\right)}}\right) c^{T} n^{*}=-\frac{d p_{e} g^{-1}\left(p_{e}\right)}{d p_{e}}\left(p_{e}\right)^{2} w\left(x_{0}\right) v\left(y_{0}\right) c^{T} v+p_{e} g^{-1}\left(p_{e}\right) v\left(y_{0}\right) c^{T}\left(\delta\left(\cdot-x_{0}\right)+(I-A)^{-1} P\left(\cdot, x_{0}\right)\right)$,

and

$$
\begin{aligned}
& D_{\Delta c_{x_{0}}}\left\|n^{*}\right\|=\frac{d p_{e} g^{-1}\left(p_{e}\right)}{d p_{e}}\left(-\left(p_{e}\right)^{2} v\left(x_{0}\right)\right)\|v\|, \\
& D_{\Delta c_{x_{0}}} c^{T} n^{*}=\frac{d p_{e} g^{-1}\left(p_{e}\right)}{d p_{e}}\left(-\left(p_{e}\right)^{2} v\left(x_{0}\right)\right) c^{T} v=\frac{d p_{e} g^{-1}\left(p_{e}\right)}{d p_{e}}\left(-\left(p_{e}\right) v\left(x_{0}\right)\right) .
\end{aligned}
$$

The formulas in (4.3) can be computed by using the fact that $\delta\left(x-x_{0}\right)$ makes sense under an integral, and both the norm $n^{*}$ and $c^{T} n^{*}$ and computed via integration. As before, the sensitivities in (4.3) are the sum 
(a)

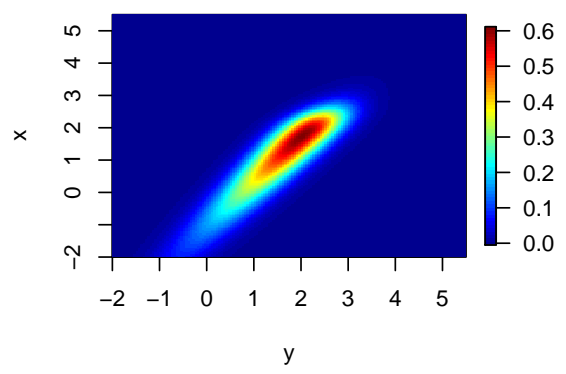

(c)

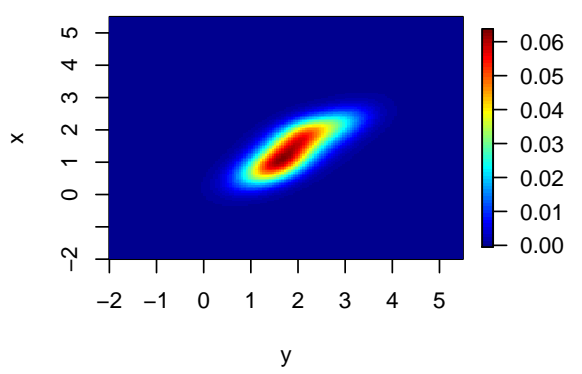

(b)

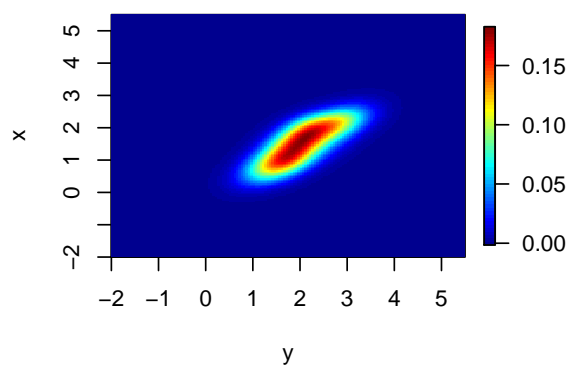

(d)

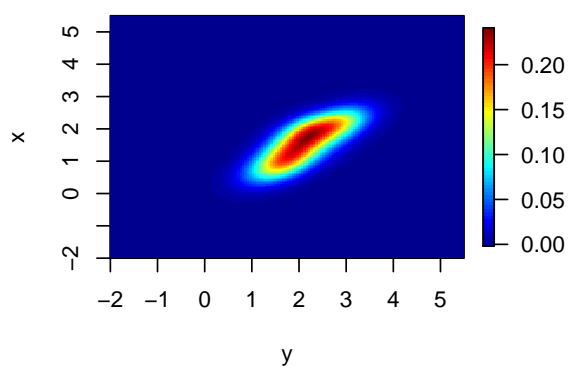

Figure 4: Illustration of the elasticity values for the equilibrium population $n^{*}$ from Rose et al. (2005) to changes in the kernel function $P(\cdot, \cdot)$. (a) gives a graph of $P(\cdot, \cdot)$, (b) the elasticities of $\left\|n^{*}\right\|$ to changes in $P(\cdot, \cdot)$ for the model using the power function, (c) the elasticities of $\left\|n^{*}\right\|$ to changes in $P(\cdot, \cdot)$ for the model using the Michaelis-Menten function and (d) the elasticities of $c^{T} n^{*}$ for the model using the power function. 
(a)

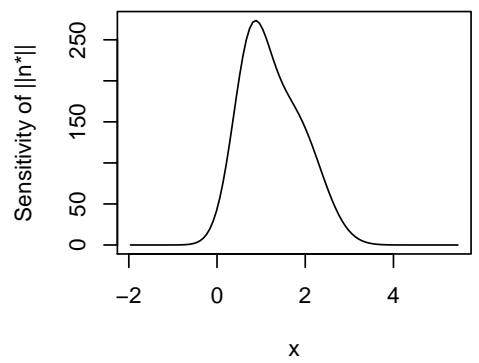

(c)

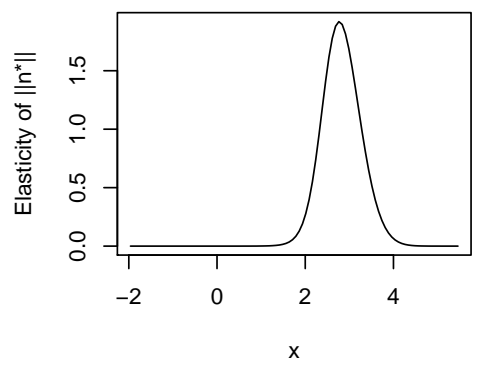

(b)

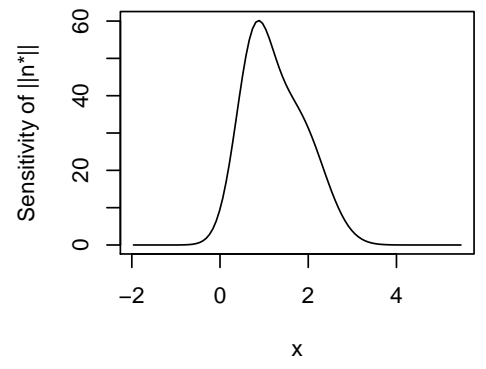

(d)

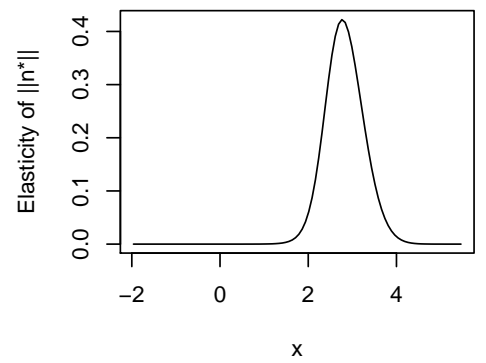

Figure 5: Illustration of the sensitivity and elasticity values for the equilibrium population $n^{*}$ from Rose et al. (2005) to changes in the function $c(\cdot)$. (a) the sensitivities of $\left\|n^{*}\right\|$ to changes in $c(\cdot)$ for the model using the power function, (b) the sensitivities of $\left\|n^{*}\right\|$ to changes in $c(\cdot)$ for the model using the Michaelis-Menten function, (c) the elasticities of $\left\|n^{*}\right\|$ to changes in $c(\cdot)$ for the model using the power function, (d) the elasticities of $\left\|n^{*}\right\|$ to changes in $c(\cdot)$ for the model using the Michaelis-Menten function. 
of the changes in equilibrium reproduction $p_{e} g^{-1}\left(p_{e}\right)$ applied equally to the entire population structure $v$ added to the specific changes in $v$ multiplied by the equilibrium reproduction $p_{e} g^{-1}\left(p_{e}\right)$. For two models that have the same equilibrium population $n^{*}$, the second term in the right-hand sides of (4.3) are identical,

and any discrepencies in sensitivity values will be elicited by different values for $-\frac{d p_{e} g^{-1}\left(p_{e}\right)}{d p_{e}}$. Fig 3 shows a heat map of the kernel function $P(\cdot, \cdot)$ and the sensitivity of the equilibrium total population $\tilde{N}=\left\|n^{*}\right\|$ for both the model using the power function for $f$ and the model using the Michaelis-Menten function for $f$. Notice that, as predicted, the qualitative features of the two plots are the same, but the sensitivities for the model with the power function are an order-of-magnitude larger than those for the model with the Michaelis-Menten function. Thus, not only does the power function elicit transient dynamics that are much more pronounced that those from the model with the Michaelis-Menten function [16], the sensitivity of the common $\| n^{*}||$ are also larger when using the power function. Notice that the hottest spots on the sensitivity figure are transitions from about $y=1$ in time $t$ to $x=5$ in time $t+1$, transitions not generally seen in the dynamics of the Platte thistle. Thus, as in the last example, we resort to the elasticity of $\left\|n^{*}\right\|$ to changes in $P(\cdot, \cdot)$ to normalize for the size of the parameter being perturbed. When looking at the elasticities, it appears as though the survival/stage transitions from about to $y=1.5$ to $x=2$ are those for which $\left\|n^{*}\right\|$ is most elastic, while some other nontrivial transitions in the life history of the Platte thistle (e.g. $y=0$ to $x=0.5)$ are those for which $\left\|n^{*}\right\|$ is relatively inelastic. Those stage transitions for which $\left\|n^{*}\right\|$ is most sensitive and elastic are the same as those for which the equilibrium total seed production $c^{T} n^{*}$ is the most sensitive and elastic (Figs 4.2 (d) and 4.2 (d) show these sensitivities and elasticities for the model using the power function, which are qualitatively the same as those with the Michaelis-Menten function).

Fig 5 (a)-(b) show the sensitivity, and (c)-(d) show the elasticity, of $\left\|n^{*}\right\|$ to changes in $c(\cdot)$, respectively. The formula for the sensitivity of $\left\|n^{*}\right\|$ with respect to $c(\cdot)$ in (4.4) shows that this sensitivity as a function of the stage variable $x$ will be shaped like $v$, the equilibrium stage distribution of $n^{*}$. However, the elasticity is not shaped like $v$, as most of the elasticity is centered on large plants, but not the largest plants. These plants have the combination of having high reproduction and a large representation in the equilibrium population, which causes this high elasticity. As with the sensitivity of $\left\|n^{*}\right\|$ and $c^{T} n^{*}$ with respect to $P(\cdot, \cdot)$, the sensitivity to $c(\cdot)$ is larger in the model with the power function than that with the Michaelis-Menten function, again showing the effects of model uncertainty on predicted population dynamics.

\section{Discussion}

In this paper we motivate and develop sensitivity formulas for the equilibrium population $n^{*}$ of a Lur'e system defined in general by (1.1). Lur'e systems have been useful in the study of both fish and plant populations subject to density-dependent feedback on reproduction and structured life-history stages. We 
show the utility of these formulas by applying them to two published population models for the dynamics of populations subject to density-dependent feedbacks in fecundity, one for a fish population and one for a plant population. A relatively simple analytical formula for the equilibrium population $n^{*}$ is known when $(A, b, c)$ and $f$ satisfy some reasonable ecological assumptions (see Theorem 2.1). This formula gives us a way to compute these sensitivities, and to interpret the two main contributions to these sensitivies: changes elicited by equilibrium reproduction $p_{e} g^{-1}\left(p_{e}\right)$ and changes elicited by equilibrium population structure $(I-A)^{-1} b$. Isolating these two factors allows us to see the differences in sensitivity values caused by different nonlinear functional forms for fecundity for the IPM in Rose et al. 38] for the Platte thistle. Specifically, using a power function for the nonlinear fecundity (which is the function that gives the smallest AIC value using the data in [38]) elicits sensitivity values that are an order of magnitude larger than when we use a MichaelisMenten function, which can be derived using first biological principles. Given that the model with the power function also predicts transient dynamics that are much larger, in many cases unrealistically larger, than the same model using the Michaelis-Menten function for fecundity, we should be skeptical of models that are statistically chosen from data without biological justification.

This work builds on existing work by [28], [4], 9], 11] and [12] in deriving the sensitivity and elasticity of equilibria for nonlinear population models, as well as that of [21] and [23] in deriving sensitivity formulas for IPMs. The novelty of this work is the rigorous construction of formulae able to handle the sensitivity of equilibria for nonlinear integral projection models as well as nonlinear matrix population models. The use of operator techniques allow us to interpret the sensitivity formulas in a natural way, showing the likely source of discrepencies in the various sensivities seen in our examples. One interesting potential extension of this work involves deriving similar equilibrium (and subsequently sensitivity) formulas for models that do not quite fit into the Lur'e formulation (1.1). These would include models for which survival/growth are density-dependent and/or there are multiple nonlinear factors that do not easily fit into in the model formulation. In [18] we explore the global stability of a coupled plant-seed bank model with two nonlinearities that do not compose in such a way as to create a Lur'e system. This is because the reproductive output of plants is subject to two density-dependent factors (seed production and seedling establishment), while the seed bank was only subject to one (seedling establishment). Despite this, the analysis in that paper revealed a formula for the equilibrium plant and seed bank population, which is globally stable in many cases, and is such that its sensitivity can be derived using the techniques in this paper. Future work will look into other models with cascading nonlinearities to see if there are any generalities that can be exploited to further our understanding of the sensitivity of the equilibrium (if such an equilibrium exists) for such models.

Another extension that we hope to consider is the sensitivity and elasticity of long-term populations coming from models with stochasticity. Most ecological systems are subject to demographic and/or environmental 
variability, and many models that incorporate this variability have populations that converge in distribution as $t \rightarrow \infty$ [3], 32], [22], [19]. A closed-form version of the probability density function for $n^{*}$ has so far proven difficult to obtain for most models, and the mean-field models for such systems are often different than one would expect due to nonlinearities in the model and Jensen's inequality. Obtaining such analytical formulas for the sensitivity and elasticity of the probability distribution for $n^{*}$ that avoid the cumbersome

simulations (as in [17], for example), would be a substantial contribution, one that will likely take analyses quite different than those in this paper.

\section{Acknowlegement}

Richard Rebarber has been partially supported by NSF grant DMS-1412598. 
(a)

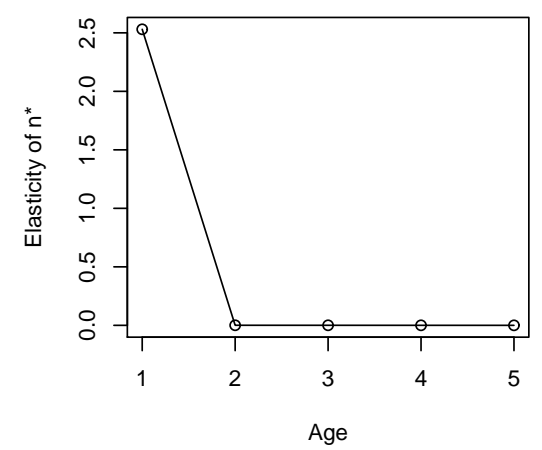

(c)

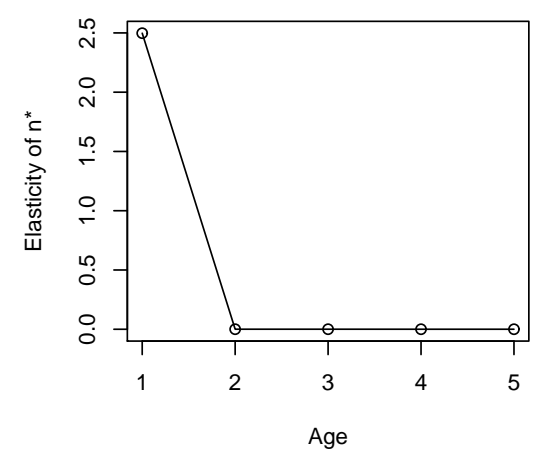

(b)

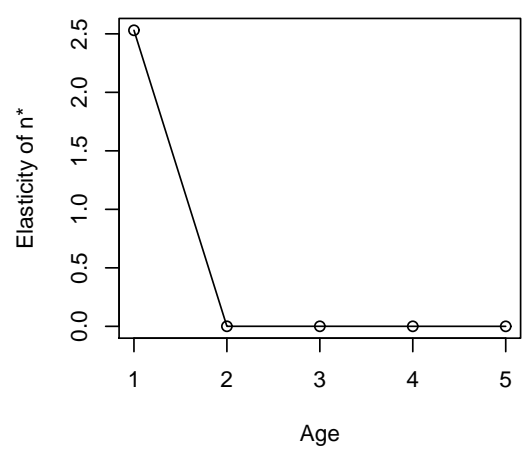

(d)

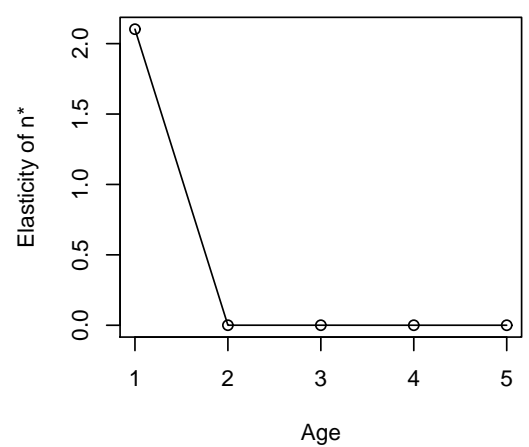

Figure 6: Elasticity values for the equilibrium population $n^{*}$ with respect to changes in the survival parameters. The horizontal axis is the age variable, while the vertical axis is the elasticity of the value of $n^{*}$ at that age variable to changes in the parameter (a) $s_{1}$, (b) $s_{2}$, (c) $s_{3}$ and (d) $s_{4}$.

\section{Appendix A: Elasticity Values for Chinook Salmon}

In Figures 6 and 7 we display the elasticity values for the Chinook Salmon model in Section 4.1

\section{Appendix B: The Sensitivity as a Distribution}

As in 23], we introduce a sequence of continuous functions $\left(\phi_{j}(x, y)\right)_{j=1}^{\infty}$ which converge in the sense of distributions to $\delta(x, y)$. Let $\mathcal{D}(\Omega)$ be the set of test functions on a set $\Omega$. The function $\phi_{j}(x, y)$ corresponds to the distribution (for $h \in \mathcal{D}\left((L, U)^{2}\right)$ )

$$
\left[\phi_{j}\right] h:=\int_{L}^{U} \phi_{j}(x, y) h(x, y) d y
$$


(a)

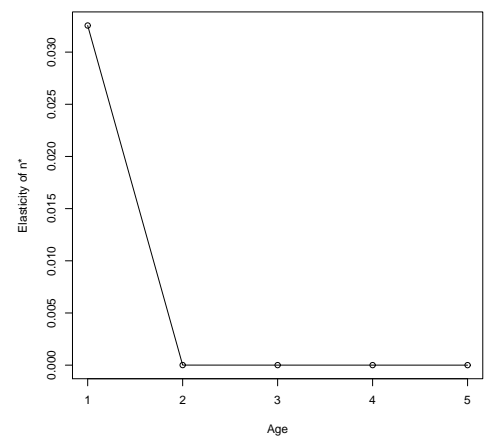

(b)

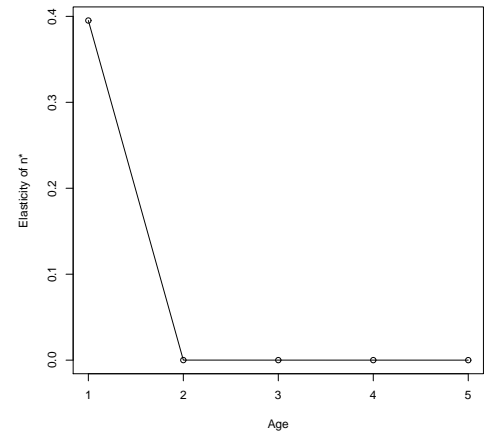

(c)

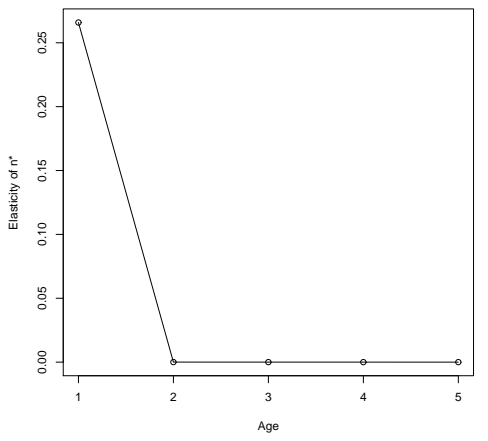

Figure 7: Elasticity values for the equilibrium population $n^{*}$ with respect to changes in the fecundity parameters. The horizontal axis is the age variable, while the vertical axis is the elasticity of the value of $n^{*}$ at that age variable to changes in the parameter (a) $f_{3}$, (b) $f_{4}$ and (d) $f_{5}$.

and

$$
\left[\phi_{j}\right] h \rightarrow h(0,0) \quad \text { for every } h \in \mathcal{D}\left((L, U)^{2}\right)
$$

For $u \in L^{1}(L, U)$, define the operators $\Delta A_{j}: L^{1}(L, U) \rightarrow C(L, U)$ by

$$
\left(\Delta A_{j} u\right)(x):=\int_{L}^{U} \phi_{j}\left(x-x_{0}, y-y_{0}\right) u(y) d y .
$$

We define

$$
\Delta A_{\left(x_{0}, y_{0}\right)}=\lim _{j \rightarrow \infty} \Delta A_{j} \quad \text { in the sense of distributions. }
$$

The approximate sensitivity of $n^{*}$ with respect to changes in $P(\cdot, \cdot)$ at $\left(x_{0}, y_{0}\right)$ is, by $(3.2)$,

$$
\left(D_{\Delta A_{j}}\right) n^{*}=-\frac{d\left(p_{e} g^{-1}\left(p_{e}\right)\right)}{d p_{e}}\left(p_{e}\right)^{2}\left(w^{T}\left(\Delta A_{j}\right) v\right) v+p_{e} g^{-1}\left(p_{e}\right)\left((I-A)^{-1} \Delta A_{j} v\right),
$$

where as before $v$ and $w^{T}$ are the left and right eigenvectors, respectively, of $A+p_{e} b c^{T}$ corresponding to the eigenvalue 1 . We define the sensitivity of $n^{*}$ with respect to changes in $P(\cdot, \cdot)$ at $\left(x_{0}, y_{0}\right)$ as

$$
S\left(x_{0}, y_{0}\right) n^{*}:=\lim _{j \rightarrow \infty}\left(D_{\Delta A_{j}}\right) n^{*}
$$

where the limit is in the sense of distributions. Using (8.1) we formally obtain

$$
S\left(x_{0}, y_{0}\right) n^{*}=-\frac{d\left(p_{e} g^{-1}\left(p_{e}\right)\right)}{d p_{e}}\left(p_{e}\right)^{2}\left(w^{T} \delta\left(x-x_{0}, y-y_{0}\right) v\right) v+p_{e} g^{-1}\left(p_{e}\right)\left((I-A)^{-1} \delta\left(x-x_{0}, y-y_{0}\right) v\right)
$$

We now give a rigorous interpretation of this formal expression.

We note that $v=(I-A)^{-1} b$ is a continuous function on $[L, U]$ : if not, this contradicts $b=v-A v$, since $b$ is a continuous function, and $(A v)$ is a continuous function of $x$, by (2.2) and the fact that $P$ is continuous 
on $[L, U]^{2}$. By a duality argument, $w$ is also a continuous function. Thus, since $\phi_{j}$ is a delta-converging sequence,

$$
\begin{gathered}
\lim _{j \rightarrow \infty} w^{T} \Delta A_{j} v=\lim _{j \rightarrow \infty} \int_{L}^{U} \int_{L}^{U} w(x) \phi_{j}\left(x-x_{0}, y-y_{0}\right) d y d x=w\left(x_{0}\right) v\left(y_{0}\right), \\
\lim _{j \rightarrow \infty}(I-A)^{-1} \Delta A_{j} v=(I-A)^{-1} \delta\left(x-x_{0}\right) v\left(y_{0}\right) .
\end{gathered}
$$

where the convergence in (8.3) is the usual convergence in $\mathbb{R}$, and the convergence in (8.4) is in the sense of distributions. We note that $\eta:=(I-A)^{-1} \delta\left(x-x_{0}\right)$ is not in $L^{1}(L, U)$ : if it was, then $(I-A) \eta=\delta\left(x-x_{0}\right)$ would be impossible, since $A$ is bounded. We can interpret $\eta$ as a distribution as follows: let $g \in \mathcal{D}(L, U)$, the set of test functions on $(L, U)$. The adjoint $A^{*}$ of $A$ is

$$
A^{*} g=\int_{L}^{U} P(y, \cdot) g(y) d y .
$$

We can use this to see that

$$
A \delta\left(x-x_{0}\right)=P\left(\cdot, x_{0}\right) \in C(L, U)
$$

in the sense of distributions (that is, $A \delta\left(x-x_{0}\right)$ is the distribution $\left[P\left(\cdot, x_{0}\right)\right]$ ). Since $\rho(A)<1$, we have $(I-A)^{-1}=I+(I-A)^{-1} A$, so

$$
\eta=(I-A)^{-1} \delta\left(\cdot-x_{0}\right)=\left(I+(I-A)^{-1} A\right) \delta\left(\cdot-x_{0}\right)=\delta\left(\cdot-x_{0}\right)+(I-A)^{-1} P\left(\cdot, x_{0}\right) .
$$

Note that this last term is a function in $L^{1}(L, U)$, and can be easily shown to be continuous in the same way that $v$ is shown to be continuous.

Using (8.2), (8.3), (8.4) and (8.5), we see that (3.6) holds in the space of distributions.

\section{References}

[1] P. Abrams, Hydra paradox: When culling animals makes them thrive. New Scientist. 27 May (2015).

[2] H. Akaike, A new look at the statistical model identification. Trans. Automat. Contr. 19(6) (1974) 716-723.

[3] M. Benaïm, S.J. Schreiber, Persistence of structured populations in random environments. Theor. Pop. Biol. 76 (2009) 19-34.

[4] A. Bill, C. Guiver, H. Logemann, S. Townley, Stability of nonnegative Lur'e systems. SIAM J. Control. Optim. 54 (3) (2016) 1176 - 1211.

[5] H. Caswell, A general formula for the sensitivity of population growth rate to changes in life history parameters. Theo. Pop. Bio. 14 (1978) 215-230. 
[6] H. Caswell, M.C. Trevisan, Sensitivity analysis of periodic matrix models. Ecology 75 (1994) 1299-1303.

[7] H. Caswell, Matrix population models : construction, analysis, and interpretation. Sinauer Associates, Sunderland, 2001.

[8] H. Caswell, T. Takada, Elasticity analysis of density-dependent matrix population models: the invasion exponent and its substitutes. Theo. Pop. Bio. 65(4) (2004) 401-411.

[9] H. Caswell, T. Takada, C.M. Hunter, Sensitivity analysis of equilibrium in density-dependent matrix population models. Ecol. Lett. 7 (2004) 380-387.

[10] H. Caswell, Sensitivity analysis of transient population dynamics. Ecol. Lett. 10 (2007) 1-15.

[11] H. Caswell, Perturbation analysis of nonlinear matrix population models. Demographic Res. 18(3) (2008) $59-116$.

[12] H. Caswell, Sensitivity and elasticity of density-dependent population models. J. Differ. Equ. Appl. 15 (2009) 349-369.

[13] T. Coulson, Integral projections models, their construction and use in posing hypothesis in ecology. Oikos 121 (2012) 1337-1350.

[14] J.M. Cushing, An introduction to structured population dynamics, conference series in applied mathematics Vol 71. SIAM, Philadelphia, 1998.

[15] J.M. Cushing, R.F. Costantino, B. Dennis, R.A. Desharnais, S.M. Henson, Chaos in ecology: experimental nonlinear dynamics. Academic Press, New York, 2003.

[16] E.A. Eager, R. Rebarber, B. Tenhumberg, Choice of density-dependent seedling recruitment function affects predicted transient dynamics: a case study with Platte thistle. Theo. Ecol. 5(3) (2012) 387-401.

[17] E.A. Eager, C.V. Haridas, D. Pilson, R. Rebarber, B. Tenhumberg, Disturbance frequency and vertical distribution of seeds affect long-term population dynamics: a mechanistic seed bank model. Am. Nat. 182(2) (2013) 180-190.

[18] E.A. Eager, R. Rebarber, B. Tenhumberg, Global asymptotic stability of plant seed bank models. J. Math. Biol. 69 (2014) 1-37.

[19] E. A. Eager, R. Rebarber, B. Tenhumberg, Modeling and analysis of a density-dependent stochastic integral projection model for a disturbance specialist plant and its seed bank. Bull. Math. Biol. 76(7) (2014) 1809-1834. 
[20] E. A. Eager, Modeling and analysis of population dynamics using Lur'e systems accounting for competition from adult conspecifics. Letters in Biomathematics 3 (1), 41-58.

[21] M. Easterling, S.P. Ellner, P.M. Dixon, Size-specific sensitivity: applying a new structured population model. Ecology 81(3) (2000) 694-708.

[22] S.P. Ellner, Asymptotic behavior of some stochastic difference equation population models. J. Math. Biol., 19 (1984) 169-200.

[23] S.P. Ellner, M. Rees, Integral projection models for species with complest demography. Am. Nat. 167(3) (2006) 410-428.

[24] S.P. Ellner, M. Rees, Stochastic stable population growth in integral projection models: theory and application. J. Math. Biol. 54 (2007) 227-256.

[25] S. P. Ellner, D. Z. Childs, M. Rees, Data-Driven Modeling of Structured Populations. Springer International Publishing, 2016.

[26] D. Franco, H. Logemann, J. Perán, Global stability of an age-structured population model. Syst. Control Lett. 65(1) (2014) 30 - 36.

[27] A. Grant, T.G. Benton, Elasticity analysis for density dependent populations in stochastic environments. Ecology 81 (2000) 680-693.

[28] A. Grant, T.G. Benton, Density dependent populations require density dependent elasticity analysis: An illustration using the LPA model of Tribolium. J. Anim. Ecol. 72 (2003) 94-105.

[29] C.V. Haridas, S. Tuljapurkar, Time, transients and elasticity. Ecol. Lett. 10 (2007) 1143- 1153.

[30] C.V. Haridas, H. Prendeville, D. Pilson, B. Tenhumberg, Response of population size to changing vital rates in random environments. Theor. Ecol. 6(1) (2013) 21-29.

[31] C.V. Haridas, E.A. Eager, R. Rebarber, B. Tenhumberg, Frequency - dependent population dynamics: effects of sex ratio and mating system on the elasticity of population growth rate. Theo. Pop. Biol. 97 (2014) 49-56.

[32] D.P., Hardin, P. Takac, G.F. Webb, Asymptotic properties of a continuous-space discrete-time population model in a random environment. J. Math. Biol. 26 (1988) 361-374.

[33] D. Hinrichsen, A.J. Pritchard, Mathematical Systems Theory I, Spring-Verlag, Berlin, 2005.

[34] W.F. Morris, D.F. Doak, Quantitative conservation biology: theory and pratice of population viability anlaysis. Sinauer Associates, Sunderland, 2002. 
[35] M.G. Neubert, H. Caswell, Demography and dispersal: calculation and sensitivity analysis of invasion speed for structured populations. Ecology 81 (2000) 1613-1628.

[36] F.X. Picó, J. Retana, Age-specific, density-dependent and environment-based mortality of a short-lived perennial herb. Plant. Biol. 10 (2008) 374-381.

[37] R. Rebarber, B. Tenhumberg, S. Townley, Global asymptotic stability of density dependent population projection models. Theo. Pop. Bio. 81 (2012) 81-87.

[38] K.E. Rose, S.M. Louda, M. Rees, Demographic and evolutionary impacts of native and invasive insect herbivores on cirsium canescens. Ecology 86(2) (2005) 453-465.

[39] D.M. Silva Matos, R.P. Freckelton, A.R. Watkinson, The role of density dependence in the population dynamics of a tropical palm. Ecology 80(8) (1999) 2635-2650.

[40] H.L. Smith, H.R. Thieme, Persistence and global stability for a class of discrete time structured population models. Discret. Contin. Dyn. S. Series. A. (DCDS - A), 2013.

[41] I. Stott, S. Townley, D. Carslake, D. J. Hodgson, On reducibility aand ergodicity of population projection matrix models. Method. Ecol. Evol. 1 (2010) 242-252.

[42] E. Symonides, J. Silvertown, V. Andreasen, Population cycles caused by overcompensating densitydependence in an annual plant. Oecologica 71 (1986) 156-158.

[43] S. Tavener, M. Mikucki, S.G. Field, M.F. Antolin, Transient sensitivity analysis for nonlinear population models. Method. Ecol. Evol. 2 (2011) 560-575.

[44] S. Townley, B. Tenhumberg, R. Rebarber, Feedback control systems analysis of density dependent population dynamics. Syst. Cont. Lett. 61 (2012) 309-315.

[45] S. Tuljapurkar, C.C. Horvitz, J.B. Pascarella, The many growth rates and elasticities of populations in random environments. Am. Nat. 162(4) (2003) 489-502.

[46] A. Verdy, H. Caswell, Sensitivity analysis of reactive ecological dynamics. Bull. Math. Biol. 70 (2008) $1634-1659$.

[47] J.M. Yearsley, D. Fletcher, C. Hunter, Sensitivity analysis of equilibrium population size in a densitydependent model for Short-tailed Shearwaters. Ecol. Model. 163 (2003) 119-129. 